\title{
Filigrane
}

Écoutes psychothérapiques

\section{À propos du narcissisme. Seconde partie}

\section{André Renaud}

Volume 20, numéro 2, automne 2011

URI : https://id.erudit.org/iderudit/1007611ar

DOI : https://doi.org/10.7202/1007611ar

Aller au sommaire du numéro

Éditeur(s)

Revue Santé mentale au Québec

ISSN

1192-1412 (imprimé)

1911-4656 (numérique)

Découvrir la revue

Citer cet article

Renaud, A. (2011). À propos du narcissisme. Seconde partie. Filigrane, 20(2), 55-85. https://doi.org/10.7202/1007611ar
Résumé de l'article

Dans le précédent numéro, une première partie présentait les théorisations successives de Freud sur le narcissisme et celles plus contemporaines de Kernberg. Dans cette seconde partie, plus clinique, l'auteur tente de démontrer un continuum entre le narcissisme normal et pathologique, d'expliquer les pathologies du narcissisme et le fonctionnement mental conséquent selon la sévérité des pathologies et des régressions. Il aborde aussi les problématiques suscitées dans les relations transféro-contre-transférentielles. d'utilisation que vous pouvez consulter en ligne.

https://apropos.erudit.org/fr/usagers/politique-dutilisation/ 


\section{À propos du narcissisme. Seconde partie}

\section{André Renaud}

Dans le précédent numéro, une première partie présentait les théorisations successives de Freud sur le narcissisme et celles plus contemporaines de Kernberg. Dans cette seconde partie, plus clinique, l'auteur tente de démontrer un continuum entre le narcissisme normal et pathologique, d'expliquer les pathologies du narcissisme et le fonctionnement mental conséquent selon la sévérité des pathologies et des régressions. Il aborde aussi les problématiques suscitées dans les relations transféro-contre-transférentielles.

\section{Le continuum narcissique}

1 y a un continuum entre le narcissisme normal et pathologique. La personnalité normale profite d'une certaine dose de narcissisme pour sa propre défense et la promotion de ses besoins et intérêts. Faute de cette dose normale, la personne se révèle timide, manque de confiance en elle-même, craint toujours de déranger, bref, son narcissisme n'opère pas comme il le devrait et les troubles s'élaborent. À l'inverse, la personne qui met continuellement de l'avant ses intérêts propres, ses goûts sans tenir compte des autres, manifeste une surestimation d'elle-même et une autre forme de narcissisme. Ces troubles peuvent être plus ou moins sévères et, dépassés un certain seuil, on parle de pathologies du narcissisme. Les troubles narcissiques de la personnalité ne constituent pas une entité homogène et cohérente, mais plutôt une sorte de kaléidoscope jouant sur plusieurs variables. Le tableau suivant, inspiré de Clarkin et al. (2006, 14-15) situe les troubles narcissiques dans l'ensemble des troubles de la personnalité. 


\section{Tableau I \\ Étendue et diversité des troubles de la personnalité}

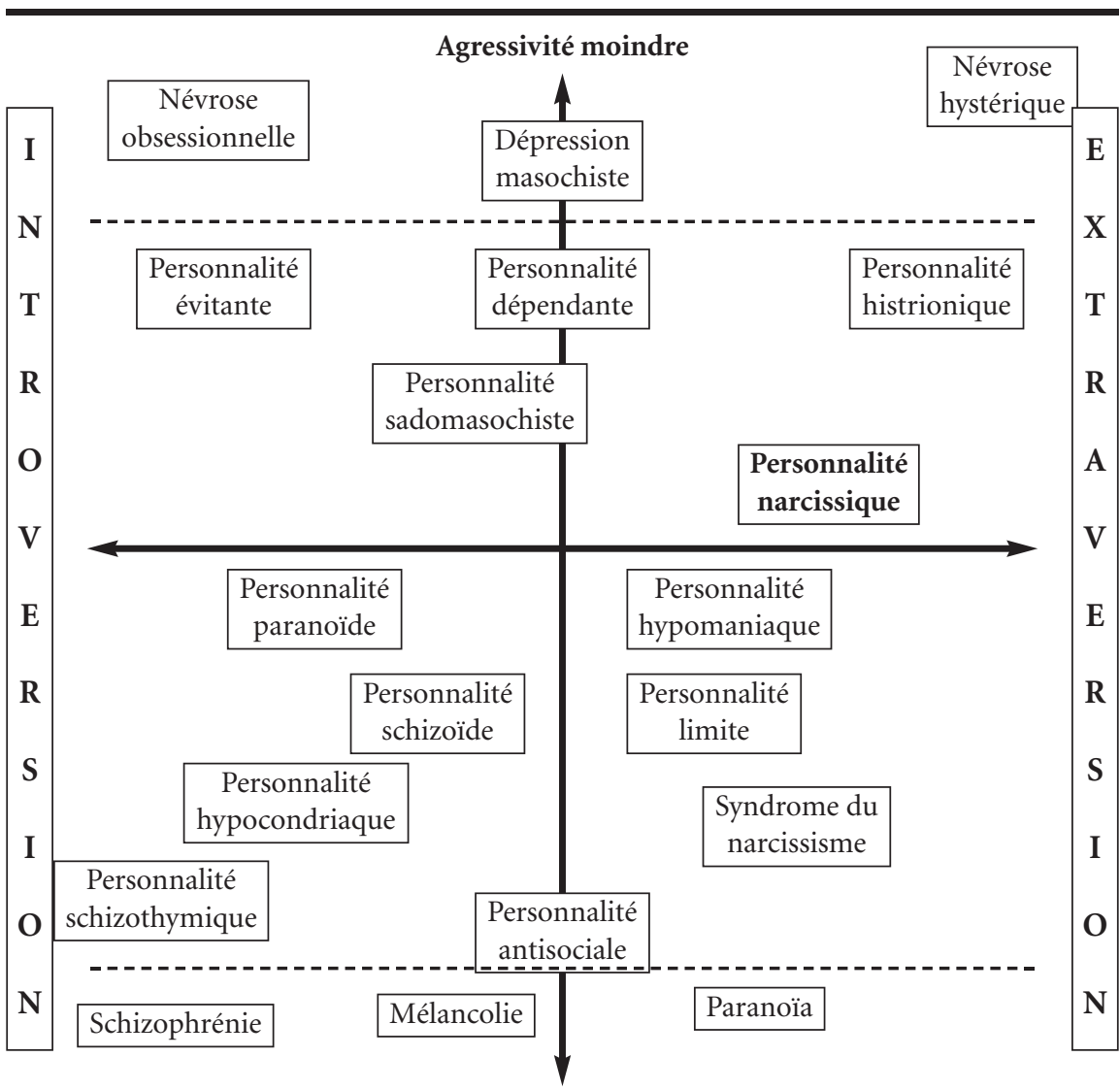

Agressivité massive et destructrice

Ce tableau implique des charges agressives moindres, tant envers soimême qu'envers autrui, dans les niveaux supérieurs et une plus grande destructivité dans les niveaux inférieurs. Les personnes dans la partie droite du tableau manifestent davantage leur caractère par leur tendance à l'extraversion, alors que celles se situant dans la gauche restent discrètes par leur tendance à l'introversion. Évidemment il faut penser un continuum entre l'agressivité moindre et la destructivité sévère, entre l'introversion et l'extraversion. Le même continuum existe à l'intérieur de chacune des cases indiquant un trouble de la personnalité, implique la présence de ce même 
continuum, le cadre de chacune indique approximativement la zone névralgique. Le trouble indiqué s'étend au-delà du cadre avec de moins en moins de spécificité et empiète plus ou moins sur les cadres voisins cernant d'autres troubles de la personnalité. Ainsi, l'étendue des troubles de la personnalité narcissique est représentée plus justement si nous précisons le continuum interne.

Tableau II

Étendue et diversité des troubles narcissiques

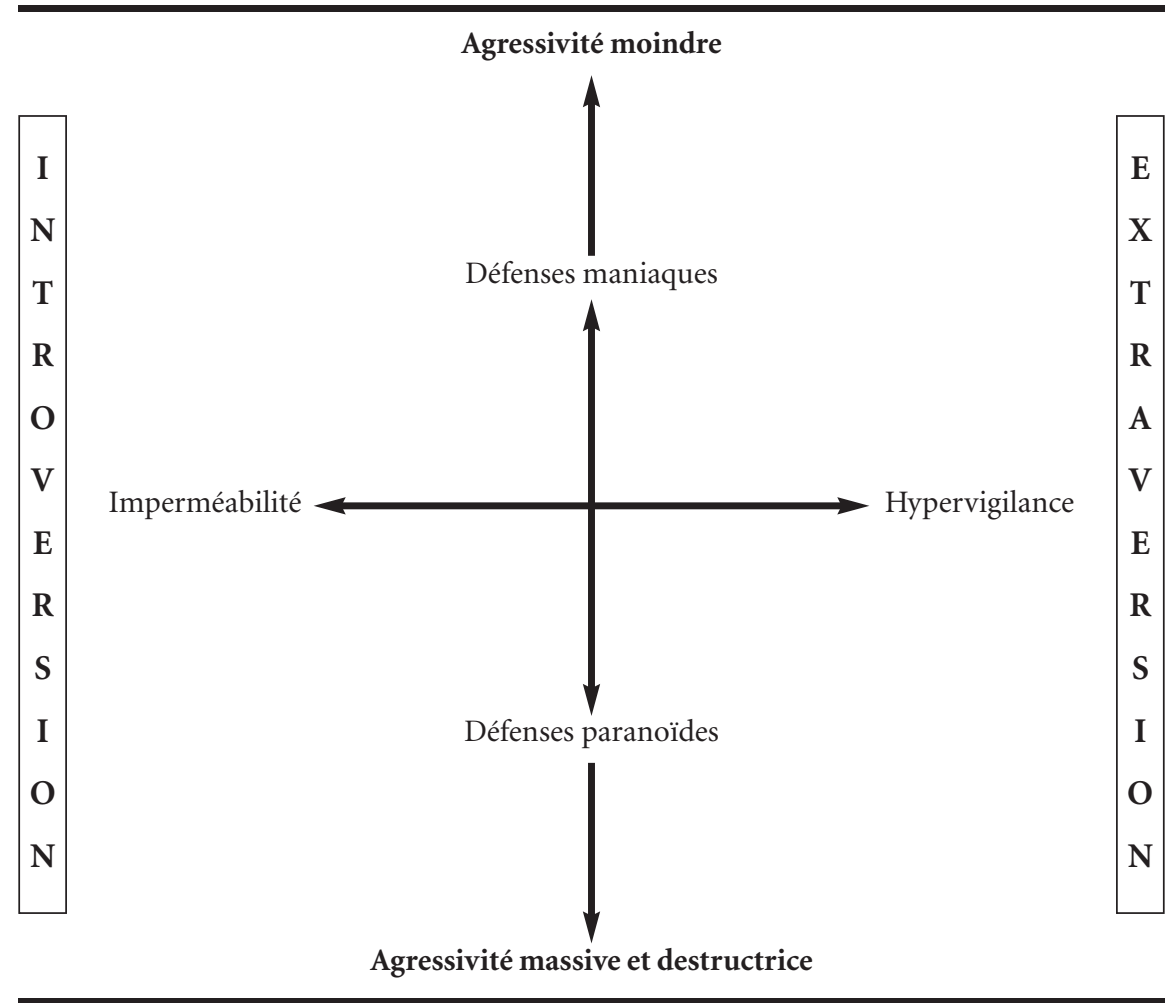

Les différences observées entre les auteurs quant à la compréhension phénoménologique et psychanalytique des relations transférentielles narcissiques reflètent les différences entre les diagnostics élaborés sur les troubles narcissiques de la personnalité. Les patients diagnostiqués «narcissiques » présentent beaucoup de différences dans leurs relations interpersonnelles et l'organisation de leur monde interne. Celles-ci dessinent un continuum de la 
vulnérabilité narcissique entre la personne imperméable apparemment fermée aux influences externes à un pôle et, à l'autre pôle, la personne hypervigilante réagissant facilement à toutes perceptions ou impressions (Manzano et al., 2005). Les patients narcissiques imperméables donnent l'impression d'une invulnérabilité à tous crins et ignorent intentionnellement les réactions d'autrui. Ces personnes sont vantardes, arrogantes, préoccupées par elles-mêmes. Elles utilisent les autres comme des spectateurs de leurs propres performances. Une défensive caractérielle ferme les protège contre les sentiments d'infériorité et de vulnérabilité narcissiques. On peut dire de ces patients qu'ils ont la peau trop épaisse (Rosenfeld, 1987) et qu'ils sont égotistes (Broucek, 1982). La relation transférentielle qui émerge en psychothérapie avec ces patients reflète bien leur style d'être. Ils peuvent passer la séance à se vanter, à se glorifier pour impressionner leur vis-à-vis, démontrer leur supériorité et ce, sans prêter la moindre attention au psychothérapeute.

Un patient narcissique arrivait régulièrement entre 10 et 15 minutes en retard à ses séances et saluait toujours son psychothérapeute par un tonitruant «Bonjour, comment ça va? » Avant que le psychothérapeute ne puisse lui rendre ses salutations, avant d'être rendu au fauteuil, il racontait les derniers évènements de son quotidien. Il fixait un point au loin devant lui, ne jetait pas le moindre coup d'œil à son hôte et disait comment il avait bien tiré son épingle du jeu dans les différentes situations vécues au travail et dans sa famille. Il parlait plus fort, ne prêtait jamais attention à ce que le thérapeute tentait de dire. Le psychothérapeute avait le sentiment de ne pas exister. Parfois, lorsque celui-ci l'interrompait en parlant par-dessus lui, il réagissait fermement: «Permettez, laissez-moi finir.» Mais il ne finissait jamais. Si le thérapeute parvenait à prendre la parole, le patient attendait impatiemment qu'il ait terminé, laissait voir son irritation et reprenait là où il avait été coupé sans prêter la moindre attention à ce qui avait été dit. Il en fut ainsi durant toute la première année de psychothérapie. Un jour, le psychothérapeute eut l'occasion de lui souligner le peu d'attention accordée aux propos de ses collègues de travail et qu'il faisait le même constat en séance avec lui. Il a répondu sèchement qu'il n'avait rien à faire des histoires des autres, il en avait déjà suffisamment des siennes. Il reprit son discours comme si le thérapeute n'avait rien dit. Il en fut ainsi encore quelques mois avant qu'une nouvelle situation donne l'occasion au thérapeute de revenir à la charge. Celui-ci devait s'absenter quelques jours. Il l'en avisa une première fois un mois avant la date, puis deux semaines et la semaine précédant son absence. Si l'avis était clair pour le psychothérapeute, il ne l'était pas pour le 
patient, puisqu'à son retour, un message sur la boîte vocale reprochait vertement au psychothérapeute son absence, son manque de professionnalisme, menaçait de porter plainte à l'ordre professionnel et exigeait un dédommagement pour son dérangement, puisqu'il s'était présenté au rendez-vous prévu. Le patient était absent au rendez-vous suivant. Le psychothérapeute lui téléphona sur l'heure du rendez-vous et lui laissa un message sur sa boîte vocale à l'effet qu'il comprenait qu'il y avait eu malentendu, qu'il importait de clarifier la situation et, à cet effet, il l'attendait à l'heure habituelle du prochain rendez-vous. Le patient s'est présenté et s'attendait à des excuses et à ne pas devoir payer cette séance, puisqu'elle était commandée par le psychothérapeute pour clarifier son erreur. Le psychothérapeute lui dit être à la fois surpris et non surpris du malentendu et de sa réaction. Il lui disait vouloir s'expliquer, mais se demandait si lui pouvait l'entendre ou si, selon son habitude, déjà relevée, il allait prêter peu d'attention à son discours et lui couper la parole à la première occasion. Le patient: "Laissez vos simagrées de côté et expliquez-vous. » Le thérapeute lui expliqua l'avoir bel et bien avisé de son absence à trois reprises, comme il l'avait fait avec tout le monde. Le patient lui coupa la parole et lui dit souffrir d'un déficit de l'attention et que c'était au psychothérapeute d'en tenir compte... Le thérapeute le regarda avec un léger sourire. Lorsque le patient aperçu le sourire, peut-être sarcastique, du thérapeute, il regarda celui-ci fixement avec défi. Le psychothérapeute lui fit remarquer qu'il lui avait coupé la parole pour prétendre un manque d'attention, mais que ce faisant, il démontrait se souvenir de l'avis d'absence reçu il y a un mois, il y a deux semaines et à la dernière séance avant l'absence :

Je comprends que vous n'ayez pas tenu compte de mon avis, parce qu'un peu comme il y a une minute, vous me coupez souvent la parole sans vraiment écouter ce que je vous dis comme si cela n'avait pas d'importance à vos yeux. Je comprends aussi que c'est votre manière de contrôler votre angoisse, votre insécurité face à ce que je vous dis, je comprends que cela fait partie de vos difficultés, de votre problème de personnalité.

Il accepta du bout des lèvres les propos du thérapeute par un "peut-être » et recommença à se défendre et à se justifier, mais avec moins de conviction.

À l'autre pôle du continuum de la vulnérabilité narcissique: la personnalité hypervigilante. La personne est extrêmement sensible aux réactions des autres, elle évite de se mettre en évidence par crainte des blessures narcissiques potentielles. Sa grandiosité est plus silencieuse et prend la forme 
d'une conviction profonde d'avoir droit à un traitement particulier, à la plus haute considération de la part des autres. Lorsque les autres semblent ne pas tenir compte de son statut spécial, ne pas la traiter avec les égards qui lui sont dus, avec la bienveillance qu'elle souhaite, alors elle se sent terriblement méprisée, blessée, humiliée. En séance de psychothérapie, elle craint à tout moment d'être humiliée, rejetée. Contrairement aux imperméables, les hypervigilants ont la peau trop mince (Rosenfeld, 1987), leur Moi-Peau (Anzieu, 2003) est troué comme un gruyère. Leur tendance au morcellement correspond à cette image.

À toutes les séances, la patiente tournait le fauteuil pivotant vers le psychothérapeute et le fixait toute la séance. Elle le surveillait, cherchait la moindre expression du visage, du regard, du corps immédiatement interprétée comme une manifestation d'exaspération, de fatigue, de rejet, de mépris en rapport avec ce qu'elle racontait, sa manière d'être, de se vêtir, etc. À la fin de chaque séance, le psychothérapeute se sentait épuisé, irrité d'être ainsi mobilisé de force, à écouter à 110 \% pour être sûr de ne manquer aucun des détails sur lesquels elle allait revenir et vérifier la qualité de son écoute et ainsi détenir la preuve de son manque de considération pour elle. Le psychothérapeute était fatigué de se sentir épié, de sans cesse se surveiller pour ne rien échapper, ne rien manifester qui ne soit pas pensé, réfléchi et jaugé. Il ne savait plus comment être.

Le thérapeute achète toutes les semaines un bouquet de fleurs coupées. Parfois la fleuriste glisse dans le bouquet une rose un peu trop ouverte pour être mêlée à un bouquet ponctuel, alors que dans ce bouquet hebdomadaire, elle l'enserre au centre du bouquet et celle-ci, ainsi retenue par les autres fleurs et le feuillage, reste belle toute la semaine. À chaque fois qu'il y a une rose dans le bouquet, la patiente la perçoit et rappelle ne pas aimer les roses; «trop commerciale». Elle a conclu que l'amour des roses du psychothérapeute traduisait son amour pour l'argent. À chaque fois qu'elle aperçoit une rose dans le bouquet, elle l'accuse de faire exprès, de ne pas tenir compte de ce qu'elle lui a cent fois répété, il ne s'occupe pas bien d'elle, il est un piètre psychanalyste qui ne comprend rien à ce qu'elle vit, etc.

Un jour, durant la saison estivale, les petits-enfants du psychothérapeute s'amusaient dans la cour arrière avec un ballon. Évidemment, le ballon roulait parfois sous la fenêtre du cabinet. À un moment donné, elle surprit le psychothérapeute à jeter un coup d'œil du côté de la fenêtre lorsqu'un enfant est venu récupérer le ballon. Il était inattentif, se plaignait-elle, il était davantage préoccupé par ce qui se passait dans le jardin que par elle, s'il lui prêtait 
un peu d'attention il réussirait peut-être à comprendre le drame de sa vie, mais puisqu'un enfant l'intéressait davantage, elle ne voyait plus ce qu'elle avait à faire dans ce cabinet. Elle s'était levée avec l'intention de quitter et d'abandonner sa psychothérapie. Le thérapeute la retint en s'excusant de sa distraction et reconnaissant que l'enfant venant sous la fenêtre le dérangeait et l'irritait parce conscient que cela l'énervait elle, que lui-même risquait d'être dérangé et que si elle s'en rendait compte, il allait devoir subir sa colère. Elle s'était rassise et lui avait ordonné de tirer les rideaux, ainsi il n'allait plus être dérangé. La séance s'était poursuivie et le thérapeute, en rappelant plusieurs situations passées, lui montrait l'exigence exagérée de l'attention centrée sur elle et sur elle seulement. Elle affirmait avoir droit à cette exigence puisqu'elle payait "plus cher pour voir un psychanalyste». Le psychothérapeute précédent lui prenait effectivement des honoraires moindres, mais les honoraires actuels étaient dans la bonne moyenne. Sans justifier ses honoraires, le psychothérapeute insista sur la revendication impérative d'en avoir pour son argent et que cela lui paraissait tout à fait justifié, mais qu'il était intéressant de comprendre pourquoi elle craignait tant de ne pas recevoir tout son dû. Après quelques rationalisations agressives, elle associa sur le fait qu'elle était la troisième d'une famille de cinq enfants, qu'elle fut vite oubliée par ses parents qui devaient s'occuper des deux derniers souvent malades, plus particulièrement le cadet né avec une malformation cardiaque alors que la quatrième souffrait d'un eczéma sévère. Elle avait toujours refusé de parler de sa famille. Elle élaborait sur son sentiment de n'avoir jamais eu de place à elle, de n'avoir pas reçu de ses parents ce qu'elle était en droit de recevoir. Une grande rage mêlée à une peine profonde a commencé à se manifester, mais cette fois, même si le psychothérapeute était encore souvent la cible de ses colères haineuses, une référence historique permettait de comprendre sa rage. Des traumas précoces pouvaient maintenant êtres explorés. Un point d'appui prenait pied, le thérapeute et la patiente pouvaient rester calmes dans un monde interne tumultueux et rageur.

Bien que le DSM-IV ne liste pas ce type d'hypervigilance, des études empiriques (Hibbard, 1992; Wink, 1991) démontrent son existence étalée sur un continum entre relativement discrète (imperméable) et évidente. Certains patients manifestent avec une certaine constante les caractéristiques propres à l'hypervigilance évidente ou à l'imperméabilité et recourent de façon aussi stable aux défenses maniaques ou paranoïdes. Cependant, la majorité des patients varient leur manière d'être entre les pôles manifestant parfois les caractéristiques propres à un pôle ou se rapprochant de ce pôle et 
parfois les caractéristiques propres à l'autre pôle ou se rapprochant de l'autre pôle. Avec la même souplesse ils utilisent les défenses maniaques ou paranoïdes selon les circonstances (Kernberg, 1974).

À cette polarisation se mêle une propension à la honte. La tendance est encore plus marquée chez la personnalité narcissique hypervigilante lorsque la réponse ne vient pas au moment souhaité et dans la forme désirée. La personnalité narcissique imperméable, fermée à l'autre se défend activement contre la honte en recherchant l'admiration, l'approbation. Cette quête de reconnaissance, de gratification est en quelque sorte un déni de l'évaluation négative du Soi liée à la honte.

\section{Les pathologies du narcissisme}

Les psychopathologies du narcissisme découlent de différents facteurs. La régulation de l'estime de Soi dépend des pressions du Surmoi-Idéal du Moi exercées sur le Moi. Plus ce système s'avère exigeant, plus les pressions morales infantiles de perfection et d'interdits restent actives et l'estime de Soi est plus difficile à conserver. Une estime de Soi faible suscite des motions agressives de la part du système Surmoi-Idéal du Moi envers le Soi et écrase ainsi les investissements libidinaux du Soi à la base de l'estime positive de Soi.

Une estime de Soi faible procède parfois d'un manque de gratifications pulsionnelles, tant libidinales qu'agressives, entraînant des élans de dépendance, de désirs sexuels et de manifestations agressives. Les frustrations pulsionnelles occasionnées par les défenses inconscientes du Moi empêchent la conscience et l'expression des motions sexuelles et agressives. Le Moi se prive alors d'expériences gratifiantes, épuise les réserves d'investissements libidinaux et diminue d'autant l'estime de Soi.

Enfin, l'intériorisation des objets libidinalement investis renforce grandement l'investissement libidinal du Soi car les représentations d'objets aimés et aimants favorisent l'amour de Soi. À l'inverse, les conflits intenses impliquent de fortes charges agressives qui ont pour effet d'affaiblir les investissements libidinaux des objets chers et des représentations intériorisées de ces mêmes objets. Par conséquent, l'investissement du Soi et l'amour de Soi en souffrent sérieusement et promptement.

\section{Trois niveaux de pathologies du narcissisme Les troubles névrotiques}

Les pathologies les plus bénignes du narcissisme sont de nature névrotique et consistent en régressions à des modes de régulation infantile de 
l'estime de Soi. C'est une perturbation relativement fréquente qui se traite bien par la psychanalyse, les psychothérapies psychodynamiques classiques et autres approches psychothérapiques.

Il y a fixation ou régression à un narcissisme infantile normal. La régulation de l'estime de Soi ou de l'investissement libidinal du Moi dépend des gratifications infantiles et de défenses contre celles-ci normalement abandonnées chez l'adulte (Kernberg, 2000). Les idéaux du Moi relèvent d'ambitions, de désirs, de valeurs ou d'interdits infantiles. Freud (1916) a décrit l'abaissement névrotique de l'estime de Soi en lien avec le refoulement excessif des pulsions sexuelles comme une des caractéristiques structurales des psychonévroses et des troubles du caractère névrotique.

La personne manifeste au quotidien des attitudes narcissiques variant du normal au pathologique. Dans certaines circonstances, elle semble narcissiquement normale, alors que dans d'autres, elle fonctionne comme une personnalité limite narcissiquement pathologique. Elle ne présente pas de symptômes névrotiques importants et fonctionne de façon relativement adaptée sur le plan social. Un peu consciente de ses difficultés émotionnelles, elle souffre principalement d'un sentiment de vide intérieur, éprouve parfois un ennui profond et manifeste un fort besoin d'approbation et de réussite. Elle se montre peu empathique et peu attachée aux autres. Certaines personnes recherchent parfois une assistance psychothérapique, mais, pour la majorité, ce sont plutôt les complications secondaires qui les conduisent à rechercher une aide psychothérapique; menace d'une rupture amoureuse, suggestion insistante de la famille, des amis, du programme d'aide aux employés, menace de perte d'emploi, etc. Lorsqu'elle se présente en psychothérapie, la personne ne tolère plus ses angoisses, ses humeurs dépressives, et ses idéations suicidaires ou tentatives de suicide inquiètent sérieusement son entourage. Ce ne sont généralement pas les troubles narcissiques qui composent le motif de consultation, mais plutôt un mal de vivre, des conflits relationnels fréquents, des sentiments d'incompréhension de la part de l'entourage, une impression d'être trop différente des autres, un sentiment de vide, d'ennui, de non-valeur, etc.

\section{Les choix narcissiques d'objets}

Des pathologies un peu plus sévères que les troubles du caractère névrotique, mais moins fréquentes, correspondent à ce que Freud (1914) appelle les choix narcissiques d'objets par rapport aux choix objectaux. Dans le choix narcissique d'objet, l'identification massive à l'objet entraîne une certaine 
confusion entre le Moi (Soi) et l'objet. Une représentation du Soi infantile est projetée sur l'objet et crée ainsi une relation libidinale infantile dans laquelle les fonctions du Soi et de l'objet deviennent interchangeables. Une partie du Moi désire être l'autre. Une partie du Moi devient l'objet et celui-ci devient une partie du Moi. Le Moi sacrifie sa propre identité en cherchant inconsciemment à être l'autre. Évidemment, l'autre ne se comporte pas nécessairement comme le Moi souhaite et, à plus ou moins court terme, ne peut que décevoir et frustrer. La privation imposée par l'autre à son insu est ressentie comme trahison, rejet, abandon, perte et en raison de la fusion-confusion Moi/autre, c'est une partie du Moi qui est perdue.

Dans le choix objectal d'un objet, l'identification s'adresse plutôt à certains traits, à certaines caractéristiques de l'autre sans entraîner de confusion aliénante du Moi ou du Soi. Le Soi et l'autre restent deux personnes indépendantes. Le Moi fait preuve d'une maturation suffisante pour éviter toute projection massive sur l'autre de ses fixations et régressions infantiles.

Les patients de ce niveau de régression narcissique présentent tous les symptômes décrits au niveau précédent, mais de façon plus marquée.

Une patiente, en analyse depuis quelques années, se dit démotivée, fatiguée de chercher à se comprendre et être déçue de son analyse. Elle espérait que l'analyste allait lui apporter une réponse manifeste à sa carence affective. Sa présence en séance, c'est pour profiter de l'accueil, des égards qui lui sont manifestés. Elle ne veut plus d'analyse et d'interprétation. Elle déplore que son analyste ne soit plus sur la même longueur d'onde qu'elle. Elle exige une reconnaissance de sa valeur, une confirmation de sa particularité, une mise en valeur de sa différence à l'égard des autres patients et patientes. Elle exige que son analyste soit avec elle, pense comme elle, soit comme elle. Elle espère que la fréquentation de l'analyste lui permette de devenir quelqu'un de bien comme lui, d'avoir son assurance, son calme, sa compréhension, son empathie. Si le changement ne s'opère pas, c'est parce qu'il est trop rationnel, trop froid, trop distant, davantage intéressé par l'analyse d'elle que par elle-même, trop centré sur la mise en évidence de son fonctionnement mental, mais pas vraiment «mordu» d'elle.

\section{Les troubles narcissiques proprement dits}

Un troisième niveau de pathologie, plus sévère encore, concerne les troubles narcissiques proprement dits, un des syndromes cliniques qui interpelle le plus les psychothérapeutes. Ce niveau compte trois grandes catégories: les troubles du narcissisme proprement dit, le syndrome du narcissisme malin 
et les personnalités antisociales (Clarkin et al., 1999). La psychothérapie de ces patients pose plusieurs difficultés et les échecs sont fréquents. Leur fonctionnement psychique implique souvent des affects intenses facilement activés, des attitudes hautaines, méprisantes et agressives, provoquant des réactions d'impatience, d'impuissance chez les psychothérapeutes.

Ces personnes sévèrement narcissiques, en dépit des défenses instaurées pour protéger le soi grandiose pathologique dans les interactions sociales, manifestent ouvertement les caractéristiques typiques des personnalités limites proprement dites. En plus des symptômes déjà décrits, mais plus marqués encore, elles manifestent un sévère manque de contrôle des impulsions, un très faible seuil de tolérance à l'anxiété et à la frustration, des capacités sublimatoires presque nulles, une tendance chronique aux explosions rageuses et aux distorsions paranoïdes.

Grâce aux nombreuses études et recherches des quarante dernières années et aux modifications apportées aux méthodes psychothérapiques pour mieux tenir compte de la nature de ces pathologies, il est maintenant possible d'engager des psychothérapies avec de meilleurs succès auprès de ce type de personnes. Les traitements psychanalytiques réussissent relativement bien avec ces personnes. Cependant, le taux d'échecs augmente drastiquement dès que les traits et les comportements antisociaux sont présents en nombre et en importance. Tant et si bien que nous pouvons considérer ne pas avoir encore de méthode psychothérapique vraiment fructueuse avec les personnalités antisociales, même si parfois certaines améliorations peuvent être enregistrées.

C'est pourquoi les prochaines pages seront consacrées aux troubles narcissiques proprement dits, plus accessibles à la psychothérapie. Trois caractéristiques marquent principalement ces pathologies: un amour pathologique pour Soi, un amour pathologique pour l'objet et une pathologie du Surmoi (Kernberg, 2000).

L'amour pathologique pour Soi s'exprime par la centration excessive sur soi et la référence constante à soi-même. Ces personnes ne parlent que d'ellesmêmes et de leurs expériences, se donnent facilement en exemple. Elles manifestent leur grandiosité en tentant d'en imposer, d'impressionner par leur récit, leur savoir-faire, leurs biens ou, au contraire, leur dénuement, leur médiocrité, leur misère, leur vide intérieur. Elles présentent des tendances exhibitionnistes, des sentiments de supériorité, de témérité même et de disproportion entre leurs ambitions démesurées et ce qui leur est réalistement possible de réussir. La grandiosité trahit les valeurs infantiles sous-jacentes; 
l'attraction physique, la puissance illimitée, la richesse spontanée et facile, la recherche excessive dans l'habillement trop chic ou trop relâché, les manières exagérées, etc. Si ces personnalités jouissent d'une intelligence supérieure, elles en font montre le plus souvent de façon prétentieuse et méprisent les autres perçus et considérés comme inférieurs.

L'amour pathologique de soi pousse la personne dans un besoin manifeste d'admiration de la part des gens autour d'elle. Autant elle recherche les compliments, l'admiration, voire l'adoration, autant elle est incapable de gratitude envers les gens qui répondent à sa quête.

Ainsi, une patiente se plaignait de l'incapacité des gens autour d'elle à vivre des relations épanouies, pleinement engagées, profondément sincères. Elle les décrivait comme égoïstes et indifférents. Progressivement, il ressortait qu'elle s'attendait à ce que les gens soient pleinement à sa disposition pour répondre généreusement à ses besoins au moment où elle les éprouvait et de la façon dont elle souhaitait qu'on lui réponde. Elle était donc facilement frustrée et fâchée et s'emballait facilement pour une nouvelle connaissance qui semblait prometteuse d'amour généreux, mais qui allait immanquablement bientôt la décevoir à son tour, puisque l'autre n'était jamais au courant du rôle de parent idéal qui lui était attribué. Si une personne tentait de répondre plus adéquatement qu'une autre, alors elle considérait cette personne prétentieuse et méprisable, pensait que cette personne ne cherchait qu'à se faire mieux voir. L'admiration, la reconnaissance, la confirmation que les autres offrent à ce type de personne sont reçues comme une chose due, allant de soi et non comme une marque appréciable ou à apprécier.

Sur le plan émotionnel, la personnalité narcissique est généralement superficielle, particulièrement dans les relations avec les intimes, s'il y en a. Cela ne signifie pas une absence de manifestations émotionnelles, au contraire, mais elles sont sans racine. La personne éprouve de façon très intense les émotions, mais elle donne l'impression de vivre l'affect pour la vibration qu'il provoque et l'excitation est relativement volatile. Le sentiment de grandiosité alterne avec les sentiments d'insécurité, d'infériorité, donnant l'impression de ne pouvoir occuper que deux positions, être supérieure à toutes les autres ou inférieure et sans valeur. La plus grande crainte est d'être une personne sans relief, sans particularité, d'être moyenne, normale, ce qui signifie être médiocre ou perçue comme telle. Le sentiment de grandiosité constitue la caractéristique la plus typique de cet amour pathologique pour soi.

L'amour pathologique de l'objet se manifeste principalement par des sentiments excessifs et envahissants d'envie beaucoup plus inconsciente que 
consciente. La personne produit moult efforts pour dénier son envie. Elle feint, tant consciemment qu'inconsciemment, une indifférence exagérée ou une dévalorisation de l'objet pour se défendre de l'envie susceptible d'être activée par l'objet. La personne narcissique est généralement consciente de son faible intérêt, ou de son manque d'intérêt pour les autres, leur travail, leurs activités, leurs goûts. Elle peut même manifester diverses intensités de mépris à l'égard des autres, de leurs occupations et activités. Inconsciemment, la personne déploie beaucoup d'énergie pour limiter ce qu'elle peut prendre des autres, cantonner les identifications, empêcher l'incorporation des objets extérieurs et, en même temps, pour dévaluer inconsciemment, voire détruire ce qu'elle désire, imite, copie, incorpore des autres.

Un jeune homme narcissique se défendait d'aimer son amie et de le laisser paraître, parce qu'il craignait qu'elle ne tente de l'exploiter, de le dominer, si elle s'en rendait compte. Il deviendrait alors vulnérable, faible, non aimable et elle le quitterait. Il en était viscéralement convaincu. Il croyait fermement qu'en jouant l'indifférent, l'exigeant, l'insatisfait, celle-ci se dévouait davantage pour lui plaire et sans jamais prendre pour acquis son amour, il lui fallait le mériter et il le lui manifestait qu'avec parcimonie.

Une autre défense contre l'envie est l'exploitation des autres, du système, des organismes, etc. L'avidité excessive de la personne narcissique suscite le désir de prendre, de s'approprier, voire de voler au besoin, ce que les autres semblent posséder et dont elle se sent privée personnellement. Elle éprouve le sentiment d'être en droit de..., d'avoir le droit à... Elle en fait une question de justice.

L'amour pathologique de l'objet rend insupportable toute forme de dépendance, même relative, dépendance relative que la personne normale accepte de bon gré lorsqu'elle en aime une autre. La personne narcissique idéalise facilement et rapidement la personne aimée, mais la dévalue et la méprise aussitôt que l'autre déçoit. Ces patients semblent inconsciemment considérer les gens autour d'eux comme des personnes exceptionnelles, des idoles, mais, généralement et assez promptement, ils les perçoivent aussi comme des ennemies qui menacent leur intégrité, réputation, image, identité ou encore comme des personnes sans valeur, insignifiantes et imbéciles. Ils sont incapables d'empathie envers ceux et celles qui les entourent, de s'engager de façon significative envers qui que ce soit. Leur intérêt pour une personne, un travail, un loisir est généralement de courte durée.

La pathologie du Surmoi n'est pas propre aux personnalités narcissiques, cependant chez celles-ci l'exigence morale est très élevée, ce qui assombrit le 
pronostic d'une psychothérapie. La pathologie du narcissisme et la perturbation affective conséquente empêchent d'éprouver de façon différenciée, divers états dépressifs. Ainsi, les divers états dépressifs sont vécus avec la même intensité, qu'il s'agisse d'un remords, d'une déception, d'une tristesse, d'une perte importante ou d'un deuil. Ces personnes dépriment facilement et massivement ou restent insensibles et imperturbables. D'autres fois, on constate de sévères sauts d'humeurs suscités par l'échec des efforts de grandiosité, des efforts pour obtenir l'admiration des autres, ou par une critique quelconque qui fracasse d'emblée la grandiosité. L'estime de Soi est davantage régulée par les sentiments de honte que par la culpabilité. Ces personnes montrent peu d'intérêt pour les valeurs éthiques, esthétiques et intellectuelles. Les valeurs sont plutôt infantiles et centrées sur la protection de l'estime de Soi, de la fierté et de la grandiosité. La dépendance excessive à l'égard de l'admiration que les autres peuvent leur manifester, à l'égard des marques d'affection et de gratification, reflète bien l'immaturité du Surmoi et son fonctionnement infantile. D'autre part, certaines personnalités manifestent une pathologie plus sévère du Surmoi qui ne s'est pas suffisamment développée pour assister le Moi dans la régulation des pulsions. Le Moi passe plus facilement à l'action, agit plus spontanément ses élans intérieurs, ses désirs, ses fantasmes sans se préoccuper de son entourage. L'autre est perçu et utilisé comme un objet à son service. Kernberg (1975) a défini ce syndrome du narcissisme malin que nous ne pouvons pas, faute d'espace, élaborer ici.

Les sentiments de vide intérieur, de solitude, d'isolement sont lourds. La personne n'est pas disposée à apprendre des autres. Si une personne tente de lui montrer quelque chose, elle en éprouve une frustration rageuse et le sentiment que l'autre l'humilie, lui démontre combien sa vie est futile, nulle, insensée et sans valeur. Elle en est très ennuyée et déprimée, alors que ses besoins d'admiration, de succès, de gratification se voient écrasés.

\section{Phénomènes transféro-contre-transférentiels et psychothérapie des troubles narcissiques de la personnalité}

Freud (1914) considérait les patients narcissiques inaptes à profiter d'un traitement psychanalytique parce qu'incapables de relation transférentielle. Cette position de Freud relève peut-être d'une réaction contre-transférentielle bien compréhensible à la manière particulière des personnes narcissiques d'entrer en relation, manière qui diffère drastiquement des personnalités névrotiques auxquelles il était davantage habitué. Les patients narcissiques paraissent si peu engagés dans le processus psychothérapique, 
dans la relation avec le psychothérapeute, que ce n'est que dans les années 1960 que l'on a compris que l'absence apparente de relation transférentielle constituait le transfert typique de ce type de patient (Brenner, 1982).

Kohut (1971) soutient que les patients narcissiques élaborent une relation transférentielle, mais l'approche interprétative classique empêche le développement de celle-ci. Le psychothérapeute doit plutôt faire preuve d'une grande empathie pour pénétrer le monde interne du patient, plutôt que d'interpréter, même avec justesse, les motivations sous-jacentes aux attitudes et comportements du patient. Celui-ci ne tolère pas que le psychothérapeute dévoile ainsi son monde interne. Le partage de ce monde intime apporte confiance et sécurité, fondements de l'alliance psychothérapique. Le patient est alors en mesure de déployer une réelle relation transférentielle. Selon Kohut et ses collaborateurs, le transfert narcissique emprunte une des formes suivantes:

1) Le transfert en miroir : Le patient cherche activement dans le regard du psychothérapeute une marque d'admiration, de validation, tout comme le nouveau-né ou le nourrisson cherche l'amour et l'acceptation de lui-même dans le regard de sa mère.

2) Le transfert idéalisant: Le patient attribue au psychothérapeute des qualités exagérées frisant la perfection.

Les besoins d'estime et de cohésion du Soi sont grands et pressants, audelà des pulsions sexuelles et agressives chez ces patients.

Ces deux premiers types de transfert reflètent l'organisation bipolaire du Soi; le Soi grandiose, à un pôle et à l'autre, l'imago parentale idéalisée. Le modèle avancé par Kohut (1977) est fondé sur les déficits du Soi et le transfert constitue un effort pour combler ces défaillances par la relation au psychothérapeute, lequel devient une extension du Soi.

3) Le transfert au jumeau: La personne perçoit le psychothérapeute comme son jumeau, un double de soi. Le Soi est projeté sur le psychothérapeute appelé à remplir les fonctions du Soi du patient.

Après le décès de Kohut en 1981, ses collaborateurs, sous l'égide de Ernest S. Wolf (1988), ont poursuivi les travaux engagés et mis en évidence deux autres types de transfert Soi-Objet.

4) Le transfert adversatif: Le patient marque une opposition au psychothérapeute par besoin d'un adversaire identifié.

5) Le transfert efficace: Ce transfert découle de celui en miroir. Le patient perçoit le psychothérapeute comme celui qui va le rendre efficient, l'amener à faire ce qu'il faut pour devenir efficace dans sa propre vie. 
Kernberg (1970) fonde sa compréhension de la relation transférentielle des personnalités narcissiques sur le modèle de la Psychologie du Moi et sur la théorie des relations objectales. Il reconnaît les transferts en miroir et idéalisants, définis par Kohut, il y perçoit plutôt une défensive visant à dissimuler l'envie, la rage et le mépris du patient envers le psychothérapeute. La projection du Soi grandiose pathologique sur le psychothérapeute en fait un être idéalisé. Le psychothérapeute n'atteint la perfection que dans le fantasme du patient, dans la réalité, il ne tarde pas à décevoir le patient par un mot, une mimique, une hésitation promptement perçue comme une manifestation de mépris, de manque de respect, de dévaluation. L'introjection du matériel projeté dans le transfert entraîne un transfert en miroir qui cède parfois à un autre type de relation objectale.

Des attitudes de désengagement et de mise à distance ne tardent pas à apparaître chez le patient. Ces défenses sont à mettre en relation avec le processus de dévaluation, de mépris envers le psychothérapeute. La relation transférentielle s'organise autour d'un noyau d'envie intense à l'égard des habiletés humaines et relationnelles du psychothérapeute à aider, à comprendre la vie, les personnes, les situations. Habiletés, capacités, talents dont le patient se sent dépourvu et qu'inconsciemment il envie. Pour se défendre d'éprouver cette envie, contribution à l'image négative de Soi et au sentiment de honte, le patient dévalue, méprise, détruit le travail interprétatif du psychothérapeute et refuse toute forme de dépendance à l'égard de ce psychothérapeute qui a trop d'influence sur lui.

L’interprétation systématique du transfert négatif se révèle être la clé de voûte du travail psychothérapique dans le modèle de Kernberg.

Une patiente en psychothérapie depuis près d'un an, menace d'abandonner si son thérapeute dit encore une seule chose blessante. Elle prétend que celui-ci ne cherche qu'à l'humilier. Un jour, elle raconte sa colère contre des collègues de travail qui ne la saluent jamais le matin, à son arrivée, comme elles le font entre elles. Elle explique:

Elles papotent entre elles, racontent leurs réactions à ce qui s'est passé dans Virginie (téléroman), parlent des films vus avec leur chum en fin de semaine, ça mémère entre elles et c'est à peine si elles me regardent quand je passe!

Elle occupe cet emploi depuis deux mois et sa faible estime d'elle-même la rend généralement très discrète et silencieuse. Le psychothérapeute lui rappelle sa timidité craintive et demande si elle ne tente pas à son insu de passer 
inaperçue. Elle se fâche vertement et lui reproche de la rendre responsable, encore une fois, de prendre parti pour les "mémères ", c'est encore elle qui n'est pas correcte.

Vous allez me dire que je suis fâchée contre vous. Oui, je le suis. J'en ai assez de me faire accuser des pires bêtises.

Après quelques minutes la tête baissée et silencieuse, elle regarde le psychothérapeute d'un air défiant. Celui-ci lui dit sur un ton calme et doux:

Vous supportez très mal, ou pas du tout, le fait que je dise des choses sur vous, des choses qui vous en apprennent sur vous-même. Même si, au fond, vous demandez cette aide, cela vous humilie et vous enrage. Il vous faut absolument rejeter, mépriser ce que je vous dis, cela vous enrage trop. Vous avez le sentiment que j'en sais plus sur vous que vous-même et cela vous parait inacceptable. C'est cette impression qui est enrageante. Je comprends et accepte cela. Cette impression vous rend jalouse en quelque sorte et votre réaction défensive est de ne rien trouver de valable et d'utile dans ce que je vous dis, de l'entendre comme une accusation injuste. Cela vous est très pénible et humiliant, mais ce que je vous dis n'est pas moins vrai pour autant, n'est-ce pas?

Elle reconnaît la justesse de la description et ajoute que la vérité n'est pas toujours facile à entendre.

L'interprétation systématique du transfert négatif est prioritaire. Selon Kohut l'idéalisation du psychothérapeute doit être acceptée à sa face même plutôt qu'interprétée. Kernberg (1974) insiste sur l'importance d'interpréter l'envie sous-jacente et le mépris que cela entraîne. Il met aussi l'accent sur la nécessité de reconnaître les sentiments positifs dans le transfert, dans le dessein que le patient ne perçoive pas les interprétations du psychothérapeute comme une condamnation de son monde interne, comme si ce monde était entièrement mauvais. Les aspects positifs des défenses narcissiques doivent être clarifiés et interprétés.

Ainsi, un peu plus tard dans la même séance, la patiente se reproche d'être «soupe au lait », s'excuse de sa colère et promet de faire effort pour ne plus être impolie. Le psychothérapeute rétorque:

Puisque ce que je dis sur vous-même sonne dans votre oreille comme une insulte blessante, comme si je vous blessais intentionnellement ou par 
manque de délicatesse, comme si je voulais avoir une emprise sur vous, vous avez bien raison de vous fâcher, vous n'êtes pas ici pour ça, d'autant que vous vous sentiez en confiance au début de la séance, en sécurité avec moi, disiez-vous. Vous vous souvenez? Ça vous demande beaucoup de courage de faire face ainsi à ces images négatives de vous-même et vous faites preuve aussi de courage en restant dans la séance malgré la difficulté. Vous qui disiez ne pouvoir faire confiance à qui que ce soit, je crois que vous faites la preuve que vous êtes plus capable que vous ne le pensez.

Dans la formulation kohutienne (1984), le transfert du Soi-Objet indique éventuellement le chemin à une relation transférentielle plus classique reliée au complexe d'CEdipe associé à la compétitivité, à la rivalité et aux représailles potentielles. Kernberg (2000) y voit plutôt un développement différent selon l'interprétation systématique des défenses narcissiques pathologiques. Le plus souvent, ce travail interprétatif aboutit à un transfert paranoïde ou à des sentiments de culpabilité et à une dépression relativement sévère, au fur et à mesure que le patient reconnaît les effets autodestructeurs de ses difficultés et projette son sadisme sur le psychothérapeute. Il devient alors paranoïde ou reconnaît son propre sadisme, s'en sent coupable, honteux, dépressif. La perlaboration essentielle de ces sentiments permet une relation transférentielle plus classique, de type névrotique. Ici, les conceptions de Kohut et Kernberg se rejoignent, mais par des moyens psychothérapiques différents.

\section{Le contre-transfert}

Les concepts de contre-transfert de Freud (1910) et d'identification projective de Mélanie Klein (1946) ont beaucoup évolué. On considère aujourd'hui une grande attention aux identifications de la part du psychothérapeute vers le patient. Racker (1957) considère l'identification comme « concordante» lorsque le psychothérapeute s'identifie à l'état affectif et subjectif et aux représentations du Soi du patient. Ce type d'identification soutient son empathie envers l'organisation interne du patient. L'identification « complémentaire » entraîne le psychothérapeute à s'identifier aux représentations d'objets désavouées dans le monde interne du patient et projetées sur le psychothérapeute activant ainsi certains conflits personnels chez ce dernier, ce qui peut l'informer sur la nature et l'intensité des conflits du patient.

Selon Ogden $(1979,1982)$ le patient désavoue et projette inconsciemment les représentations du Soi et de l'objet sur le psychothérapeute dans le 
dessein que la pression ainsi créée contraigne celui-ci à s'identifier à ce qui a été projeté sur lui. De cette façon, l'identification projective peut être vue comme un précurseur de l'empathie qui aide le psychothérapeute à comprendre ce qui se passe dans le monde interne du patient. Ainsi, le psychothérapeute contient un temps les projections du patient, puis exécute un travail d'élaboration psychique sur ce matériel avant d'en interpréter le contenu au patient, ce qui, ultimement, instaure une nouvelle forme de relation objectale entre le patient et le psychothérapeute, relation qui peut éventuellement être intériorisée par le patient.

Le plus souvent, l'expérience contre-transférentielle est donc partiellement déterminée par les relations et conflits personnels et, tout aussi partiellement, par ce qui est maintenant activé chez lui par les projections du patient. À cet égard, le contre-transfert résulte d'une création commune des deux protagonistes. En fait, le psychothérapeute porte une attention soutenue à son contre-transfert afin de l'analyser et de l'utiliser aux fins de la psychothérapie et du mieux-être du patient.

Cette façon de comprendre le contre-transfert fournit une information pertinente tant sur les conflits du patient que sur ceux du psychothérapeute. Le patient narcissique a tendance à considérer le psychothérapeute comme le prolongement de son propre Soi et se perçoit, par conséquent, en mesure d'activer certains états chez le psychothérapeute reflétant en miroir ses luttes internes, ses conflits, ses états d'âme. Évidemment, l'histoire personnelle, le caractère et le tempérament des psychothérapeutes les incitent à réagir différemment aux tensions du monde interne des patients.

Certaines formes de réactions contre-transférentielles sont plus fréquentes. Elles ne sont pas exclusives aux psychothérapies des patients narcissiques, mais on les retrouve plus facilement et indéniablement avec ces patients. La réaction contre-tranférentielle la plus fréquente est la négation de son existence. Il faut un certain temps et une solide relation de confiance en supervision professionnelle avant que le psychothérapeute accepte de parler ouvertement de ses réactions personnelles à la manière d'être du patient, à son histoire et à ses provocations. D'autres réactions contre-transférentielles sont plus subtiles et plus difficiles à détecter.

Une avenue contre-transférentielle qui guette la majorité des psychothérapeutes est l'ennui (Gabbard, 1998). Le psychothérapeute trouve le temps long, s'endort, se sent tenu à distance par le patient, ne pas exister, n'avoir aucune importance. Kernberg (1970) décrit cet état: «Le psychothérapeute a le sentiment d'être un satellite du patient ${ }^{1}$.» Les professionnels en santé 
mentale ont besoin de se sentir utiles, que leur aide soit désirée, mais les patients narcissiques sont généralement frustrants sur ce point. Plusieurs patients, particulièrement ceux qui se rapprochent du pôle imperméable, parlent sans arrêt sur un ton parfois monotone, ou restent silencieux, circonspects, méfiants. Ils établissent rarement un contact visuel. Ils parlent sans pour autant s'adresser au psychothérapeute qui développe le sentiment d'être utilisé comme un magnétophone et non comme une personne. Ne pas se sentir considéré peut devenir ennuyeux et ennuyant à un niveau intolérable.

Le développement de la relation transféro-contre-transférentielle reflète, jusqu'à un certain point, l'inhabileté du patient narcissique de dépendre de quelqu'un pouvant lui venir en aide. Il se défend activement contre l'envie sous-jacente de posséder les mêmes qualités que le psychothérapeute et faute de les éprouver pour lui-même, tente de les dévaluer, de les mépriser chez son vis-à-vis. L'identification projective fait en sorte que le psychothérapeute se sent mis à distance et exclu par le patient de la même manière que ce dernier a été mis à distance et exclu lorsqu'il était enfant (Adler, 1986). Le vécu infantile est reproduit dans le transfert et le patient tente de maîtriser activement son expérience infantile traumatique en le faisant vivre au psychothérapeute.

Si ce renversement des rôles peut devenir conscient au psychothérapeute, celui-ci peut utiliser de façon constructive son contre-transfert et interpréter le renversement de la situation vécue enfant au patient:

Je me demande si, jusqu'à un certain point, vous n'essayez pas de me tenir à distance de la même manière que vous vous sentiez, enfant, tenu à distance par vos parents, comme si c'était votre façon de me faire comprendre ce que vous avez vévu. Vous voyez de quoi je parle?

Le psychothérapeute doit être conscient de ses propres traits narcissiques pour faire une telle interprétation, parce que l'ennui contre-transférentiel peut déborder le besoin du psychothérapeute d'être important pour le patient.

Il n'est pas rare de rencontrer, dans les motivations du psychothérapeute à pratiquer son métier, un désir d'être idéalisé, aimé, utile (Finnel, 1985). Le psychothérapeute peut éprouver des sentiments tout à fait contraires; se sentir inutile, incompétent, impuissant, insignifiant. Au fur et à mesure que de tels sentiments blessent narcissiquement le psychothérapeute, ce dernier peut s'en défendre en les attribuant projectivement au patient, comme s'il n'était 
pas concerné par ces affects. Un grand malentendu s'installe entre le patient et le psychothérapeute.

Il arrive parfois que le psychothérapeute soit conscient de son idéalisation par le patient. Il est alors possible que des sentiments de culpabilité et de honte surgissent, parce qu'il éprouve un certain contentement d'être ainsi perçu, se détend, prend ses aises et reste passif un temps pour ne rien déranger et tirer un peu profit de la situation. D'autres se défendent promptement de toute idéalisation, ne supportant pas pareil transfert par crainte de trop promptement décevoir, de se révéler en deçà des compétences et des qualités attribuées. Ceux-ci interprètent prématurément l'idéalisation afin de lier leur propre anxiété (Kohut, 1984). D'autres ont de la difficulté à composer avec leur propre agressivité et, tout comme les patients, tentent de parer à toute hostilité en favorisant l'idéalisation.

Un psychothérapeute avait autorisé le patient à lui téléphoner n’importe quand en cas de besoin, dans le dessein conscient que le patient le perçoive comme le bon père disponible qui lui avait tant manqué. En supervision, il fut mis en évidence qu'une telle autorisation stimulait l'envie du patient à l'égard de sa bonté, générosité, disponibilité et nourrissait une haine rageuse.

Une réaction transféro-contre-transférentielle particulière se déploie avec les patients narcissiques hypervigilants (Gabbard, 1994). Le patient interprète parfois tout mouvement du psychothérapeute comme une preuve de désintéressement, de rejet. Le psychothérapeute peut développer l'impression d'être contrôlé par son patient. Cette impression se traduit par une crainte que s'il tousse, boit, regarde l'heure, croise ou décroise une jambe, bouge sur son fauteuil, quitte le regard de son vis-à-vis quelques secondes, etc., il provoquera une crise, une colère qui dévastera le patient dans son estime de Soi. De la même manière, le psychothérapeute peut être conscient que le patient hypervigilant tente ainsi de le contrôler dans sa manière de penser, de dire, d'être, mais l'impression d'être contrôlé persiste, le sentiment d'avoir perdu sa liberté reste marqué. L'identification projective peut être interprétée comme un effort de la part du patient pour contrôler la liberté de penser du psychothérapeute. Celui-ci se sent confiné à une marge relativement étroite de pensée, d'associations et d'utilisation de mots (Symington, 1990). Il se sent bousculé, harcelé par l'interdit posé par le patient de ne parler que de certains sujets et de rien d'autre. Lewin et Schulz (1992) recourent à une image bien représentative de ce type de relation transféro-contre-transférentielle; le patient est le ventriloque et le psychothérapeute est la poupée. 
Une patiente dans la cinquantaine, après deux ans de psychothérapie, se dit très déçue de son psychothérapeute. Elle lui reproche de manquer d'empathie envers sa misère. Elle entreprend, pendant plusieurs séances de lui montrer comment travailler avec des patientes en difficulté comme elle. Elle lui enseigne à être empathique et ce qu'il faut dire et comment le dire, avec quelle intonation, sur quels mots insister, lui donner le temps et le droit de réagir, etc. Elle insiste. Après quelques séances, le psychothérapeute comprend que ce qu'elle cherche et lui demande d'être, c'est la mère idéale qu'elle n'a pas connue, une mère qui anticipe les besoins de son enfant, qui sait la consoler et la rassurer lorsqu'elle se blesse et non lui montrer comment elle s'est blessée elle-même. Une mère qui donne tellement d'amour et d'attention à sa fille que celle-ci n'a pas besoin de le quêter. Le psychothérapeute reconnaît pour lui-même qu'il lui est difficile de tolérer ces séances d'enseignement extrêmement méprisant et que l'agressivité qu'il éprouve lui en apprend sur l'envie et la rage de cette patiente. Après quelques séances de ce traitement pénible, il lui devient clair qu'il doit clarifier la situation. Plus elle lui enseigne, plus elle devient exigeante et méprisante. Il craint ne plus pouvoir contenir son irritation et exprimer quelque chose avec un peu d'irritation et d'impatience, confirmant la patiente dans son sentiment de ne pas être aimée, de ne pas l'intéresser. Il reprend une phrase utilisée par la patiente en début de séance:

Votre enseignement sur la bonne façon d'intervenir auprès de vous et des autres femmes dans votre situation est très précieux, en fait il me fait mieux comprendre ce que vous disiez en début de séance aujourd'hui, que lorsqu'une enfant n'a connu qu'une mère dépressive et anxieuse vingt-quatre heures sur vingt-quatre, cet enfant a effectivement gravement manqué d'attention, d'affection, de sécurité, comme vous disiez. Cependant, je me demande si ce que vous me demandez d'être avec vous ne ressemblerait pas à cette mère que vous n'avez pas eue...

Elle lui coupe la parole :

Mon dieu, commenceriez-vous à comprendre enfin...

Le psychothérapeute reprend:

Je l'espère pour vous, mais $j$ 'ai aussi le sentiment de comprendre autre chose et peut-être pourrez-vous ajouter à mon propos. Je me demande s'il vous serait possible de recevoir les soins d'une aussi bonne mère. Je m'explique si vous voulez bien. Je me dis qu'il a été tellement souffrant d'avoir une mère aussi insuffisante que, d'une certaine façon, ça doit être inacceptable. Je me 
demande s'il est possible à une personne de reconnaître que sa mère ait été aussi insuffisante, je crains que ce ne soit trop souffrant, on espère toujours qu'elle a fait au moins son possible...

Elle lui coupe la parole :

C'est ce que je vous dis, c'est trop souffrant, c'est inacceptable.

Il continue:

Voilà, je pense que nous disons la même chose. Ma question est la suivante: Si une meilleure mère se présentait pour prendre soin d'une enfant aussi délaissée, ne ferait-elle pas sentir à cette enfant tout ce qu'elle a manqué, ne lui ferait-elle pas mal de lui montrer tout ce qu'elle a manqué, est-ce que ce serait supportable, ne lui ferait-elle pas plus de mal que de bien.

Elle le regarde d'un air interloqué, surpris. Il poursuit:

Vous savez, les gens qui font une grève de la faim, qu'on retrouve après vingt, trente, quarante jours de jeûne, ces gens ont tellement faim qu'ils ne sentent plus la faim et si on leur donnait tout de suite un bon repas normal, on les tuerait, tellement ils ont des manques multiples dans leur organisme après un tel jeûne. Vous comprenez ce que je veux dire?

Elle le regarde et pleure doucement. Il continue:

Alors, je me demande si ce n'est pas de ce genre de souffrance dont vous souffrez ici avec moi.

Elle le regarde calmement et dit:

Peut-être. Ça fait tellement mal en dedans. Je suis vide.

Il ajoute:

Mais vous êtes là et je suis là, nous sommes maintenant ensemble dans cette souffrance, vous avez réussi à me la faire comprendre, du moins je pense. Qu'en dites-vous? Est-il possible pour vous de poursuivre votre psychothérapie ici avec moi, même si ça fait mal, même si ça réveille de vieilles blessures pénibles?

Elle le regarde fixement et dit:

Ne me laissez pas tomber même si je suis détestable.

Il ajoute:

C'est ce qui vous fait peur.

Un autre phénomène contre-transférentiel déconcertant et régulièrement observé dans les psychothérapies de patients narcissiques est la réaction au mépris et aux sarcasmes du patient (Gabbard, 1994). Certains patients éprouvent le besoin de rappeler régulièrement au psychothérapeute que son discours n'est pas nouveau et est inutile. Ils affirment ouvertement que les 
informations apportées par le psychothérapeute sont déjà connues et qu'ils ne voient pas en quoi celui-ci peut prétendre l'aider.

Un patient a fait comprendre cela de belle manière à son psychothérapeute. Celui-ci venait de lui faire remarquer poliment que s'il avait manifestement traité son collègue de travail de la façon dont il venait de le rapporter, qu'il y avait dans sa remarque une teinte suffisante de mépris qui expliquait peut-être la réaction fort négative du collègue. Sans lui parler directement, mais plutôt en soliloquant le patient répondit:

Pis ça a été professeur d'Université c't'affaire-là. Vraiment, j'ai pas de complexe à avoir avec ma cinquième année $b$ !

Il s'était senti accusé, méprisé à son tour par la remarque du psychothérapeute.

Ces patients craignent terriblement de se sentir honteux, humiliés ou envieux et s'en défendent très activement. Ils traitent parfois le psychothérapeute avec mépris, lui reprochent un attachement rigide à son rôle professionnel, d'être incapable de sincérité naturelle et spontanée. Son rôle lui sert de costume protecteur pour se sécuriser, se donner confiance et contenance, bref, le psychothérapeute est considéré plus malade que ne l'est le patient.

Une patiente, au début de la trentaine et fort jolie, avait reçu de son psychiatre le diagnostic de personnalité narcissique avec de forts traits histrioniques et la recommandation d'engager une psychothérapie. Elle manifestait aussi quelques traits pervers et quelques conduites antisociales. Elle vivait, ou plutôt se faisait vivre par un homme d'affaires de 20 ans son aîné. Elle l'exploitait littéralement. Elle avait régulièrement des aventures avec d'autres hommes de son âge et même plus jeunes qu'elle. Elle aimait séduire, provoquer, jouer à la femme fatale et exhibait ses charmes avec un plaisir évident. Un jour, le psychothérapeute a dû lui souligner qu'elle semblait confondre la situation de psychothérapie avec celle d'un bar de rencontres cherchant, par sa manière d'être, à dévier le but des rencontres. Elle s'est fâchée, l'a traité de straight, de soumis à sa petite femme chérie, de castré, de pédé, etc. Il lui a dit comprendre qu'elle craignait son monde interne et, pour cette raison, elle tentait de détourner son attention vers ses charmes plutôt que d'explorer son monde intérieur, mais qu'il lui était difficile d'oublier qu'elle était aussi et surtout une personne en difficulté, malheureuse dans sa peau, une personne qui espérait son aide. L'interprétation fut sans effet. Un autre jour, dans une confrontation plus musclée, elle lui reprochait de se cacher derrière un rôle 
professionnel parce qu'il devait sans doute souffrir d'impuissance sexuelle, elle ne voyait pas d'autre raison parce que jamais un homme ne l'avait refusée, seul un psychanalyste homosexuel pouvait ainsi refuser ses «avances flatteuses».

À la séance suivante, elle apporte deux cafés et deux muffins en disant sur un ton mi-blagueur, mi-provocateur :

Les homos, ça refuse les femmes, mais ça ne refuse pas un bon café...

Agacé, irrité, le psychothérapeute lui a répondu qu'ils n'étaient pas chez Tim Horton et qu'ils ne se rencontraient pas pour prendre un café et socialiser. Elle s'est évidemment fâchée ouvertement, l'a traité de tous les noms avant de conclure qu'elle perdait son temps et son argent avec un homme aussi «straight et plate, aussi poigné par en-dedans». Sur ce, elle s'est levée pour partir. Il lui dit comprendre que sa réaction agacée était certes agressive et offensante et s'en excusait, que sa colère lui faisait comprendre toute la charge agressive et haineuse qu'elle éprouvait à l'égard des hommes en général, que chacun d'eux représentait un ennemi qu'elle devait allonger pour l'abattre, comme on abat un arbre en l'allongeant sur le sol. Elle l'a regardé d'un air ahuri et lui a lancé sur un ton rageur:

Vous saurez que les hommes, c'est moi qui les baise, il n'y en a pas encore un qui m'a baisée!

Il lui a répondu promptement:

C'est bien ce que je dis, vous êtes davantage engagée dans une histoire de haine que dans une affaire amoureuse!

Elle est restée figée, silencieuse un moment et, hésitante, lui a dit qu'elle n'avait jamais vu les choses sous cet angle. Elle s'est assise. Il a tout de suite ajouté que justement c'était ce qui la déconcertait ici, il refusait de faire la guerre, de faire la haine et qu'il l'aimait suffisamment, telle qu'elle était, pour tenter de l'aider si elle voulait bien de son aide. Elle a poursuivi le processus et ses tentatives de faire dévier ont pris d'autres formes moins dérangeantes, comme les honoraires et les reçus. La résistance a tenu encore un temps avant de diminuer progressivement et un travail plus en profondeur a pu lentement s'engager. 
Les situations ne s'arrangent pas toujours aussi bien. Parfois, la personne insulte plus ouvertement et plus rageusement encore le psychothérapeute, lui manifeste vertement un tel mépris que ce dernier ne peut plus en supporter davantage et pense abandonner le processus et lui proposer consulter un autre collègue. D'autres fois, le psychothérapeute tente de se débarrasser du patient en lui témoignant, souvent à son insu, du rejet, en s'enfermant dans un mutisme feignant l'indifférence, tant et si bien que le patient met les voiles. D'autres psychothérapeutes ripostent de façon sarcastique, parfois même sadique et le patient n'en pouvant plus quitte le processus.

Ce type d'interaction mine parfois la confiance du psychothérapeute en lui-même et lui fait éprouver un sentiment d'impuissance assez désagréable. Ces réactions contre-transférentielles échappent le plus souvent à la conscience du psychothérapeute qui répète alors le même genre d'interactions que le patient a vécu jadis avec ses propres parents, intériorisés et remis en scène dans la relation transférentielle. Autrement dit, le patient cherche à se faire rejeter, abandonner en incitant le psychothérapeute à agir de la sorte par diverses attitudes et conduites. Être un objet durable capable de contenir les agressions, la haine, l'envie, le mépris est sans doute la voie psychothérapique par excellence en pareil moment, mais cela est nettement plus facile à conseiller qu'à appliquer personnellement et professionnellement. Si le psychothérapeute peut parvenir à contenir son irritation, il peut utiliser son contre-transfert et tenter une interprétation.

Certains patients narcissiques tout à fait charmants provoquent parfois une réponse en miroir chez le psychothérapeute coïncidant avec le fantasme d'un objet interne admiré, confirmé et aimé inconditionnellement. Le psychothérapeute peut consciemment mettre l'accent sur le besoin de son patient de se mirer, mais inconsciemment faire collusion et collaborer à son insu à une relation transféro-contre-transférentielle qui exclut toute forme d'agressivité et tout autre affect négatif. Le patient semblant s'engager dans la relation, le psychothérapeute se retrouve alors pris et abandonne, sans s'en rendre compte sur le champ, sa fonction de psychothérapeute au profit d'une situation d'un contentement mutuel d'être ensemble (Gabbard, 1998). Ce qui peut conduire les deux protagonistes à une admiration mutuelle excluant le reste du monde, un monde dans lequel le patient connaît pourtant de nombreuses difficultés interpersonnelles. Pendant que l'admiration réciproque s'élabore et se maintient, le reste de l'organisation de vie du patient se détériore. Le psychothérapeute manifeste de l'empathie envers la position de victime présentée par le patient, alors que ce même patient en dehors des séances 
de psychothérapie abuse sadiquement ceux et celles qui l'entourent et fait d'eux et d'elles de vraies victimes.

Un supervisé raconte comment son patient dans la mi-trentaine est maltraité par sa famille dont les membres sont tous des professionnels de carrière et financièrement à l'aise. Cependant, les deux frères et la sœur refusent de lui venir en aide. En tenant compte de l'intonation du supervisé, le superviseur supute un parti pris en faveur du patient contre la mauvaise famille et demande au psychothérapeute si le patient travaille actuellement. Non, il est étudiant. Il n'a jamais travaillé. Pour quelle raison? Il n'a pas eu la possibilité de travailler, il a toujours étudié, mais c'est là sa difficulté. Il a changé plusieurs fois de programmes d'études, éprouve de la difficulté à trouver sa voie. Il n'a jamais terminé un programme universitaire parce qu'il se lasse et abandonne. Le superviseur pose plusieurs questions et apprend, en même temps que le psychothérapeute prend conscience de la situation globale, que le patient habite gratuitement le logement de son frère aîné, possède une voiture neuve payée par ses parents, profite d'un ordinateur portable, cadeau de sa sœur, qu'à son dernier anniversaire son autre frère lui a offert un voyage en Europe, son épicerie hebdomadaire est achetée par la mère, les soldes mensuels de sa carte de crédit sont assumés par le père, il a des relations sexuelles avec plusieurs amies, consomme relativement régulièrement quelques drogues, etc. Le superviseur fait remarquer au psychothérapeute avoir déjà rencontré des gens plus maltraités. Il ressort que ses frères et sa sœur ne lui donnent plus d'argent comptant parce que celui-ci lui coule entre les doigts. Ses parents ont réduit la limite de sa carte de crédit à mille dollars parce qu'il dépassait régulièrement le plafond de crédit fixé. Plus récemment, on a fermé la marge de crédit à sa banque parce qu'il n'est plus en mesure de la rembourser. Il refuse de travailler comme assistant de cours ou de laboratoire parce qu'il est trop angoissé de faire face aux tâches qu'on lui confie. On comprend que la famille tente de gérer au mieux les finances du petit dernier qui semble faire la vie de pacha en exploitant tout le monde. Et encore, le psychothérapeute n'a pas exploré l'exploitation potentielle des $\operatorname{ami}(\mathrm{e}) \mathrm{s}$.

Une difficulté créée par l'acceptation inconditionnelle excessive et contretransférentielle du psychothérapeute face au patient narcissique est que ce dernier croit cette acceptation tout à fait méritée et qu'il est en droit d'attendre pareille acceptation des autres. Il considère cette acceptation inconditionnelle du psychothérapeute comme le signe qu'il n'a pas à changer son monde interne, son mode de vie, sa manière d'être. Il n'a qu'à trouver, dans 
le monde externe, le ou la partenaire de vie qui, comme le psychothérapeute, l'acceptera pleinement et lui apportera toutes les satisfactions.

Une façon de sortir de ce cercle vicieux est évidemment encore une fois de prendre conscience et d'utiliser la réaction contre-transférentielle. Prisonnier de cette admiration réciproque, il est difficile au psychothérapeute de la réaliser et, le plus souvent, c'est en supervision qu'il s'en rend compte. C'est pourquoi il est recommandé de ne pas travailler de façon isolée avec ce type de patient. Le psychothérapeute a besoin de discuter professionnellement de son travail avec un collègue. Ayant vécu ce type de situation avec un patient et ayant discuté avec un superviseur de son travail qui stagnait, un psychothérapeute a pu ainsi se défaire d'une admiration réciproque qui faisait stagner le processus en rappelant au patient les motifs de sa consultation énoncés dans les rencontres préliminaires et lui soulignant qu'il n'en parlait plus au profit d'un discours bienséant mais éloigné de ses difficultés. Ce faisant il lui était difficile de le comprendre et de lui venir en aide puisqu'ils parlaient de tout autre chose au lieu de parler de ce qui l'incitait à consulter.

\section{En guise de conclusion}

La majorité des psychanalystes et des psychothérapeutes psychanalytiques ne travaillent pas avec une adhésion parfaite et rigide aux modèles psychothérapiques (Gabbard, 1994). En pratique, les psychothérapeutes font preuve d'une plus grande flexibilité que les modèles ne le prévoient et que les formateurs ne la tolèrent. Le problème rencontré en clinique avec les modèles est la tentation de forcer une personne à correspondre à une classification rigide, d'exiger que le patient s'adapte à un modèle plutôt que le psychothérapeute et son modèle ne s'adaptent au patient, sans pour autant devenir esclave du patient. Les modèles sont utiles pour aider à réfléchir, à comprendre, à formuler des hypothèses explicatives, mais ils ne remplacent pas la personne du psychothérapeute et celle-ci n'est pas un robot préprogrammé pour appliquer mécaniquement un modèle. Même dans l'application des modèles les plus structurés, les plus manualisés, les psychothérapeutes sont constamment obligés de faire des adaptations, de tenter des procédures imprévues. La personnalité du psychothérapeute, sa sensibilité, sa créativité et son expérience cliniques sont meilleures conseillères que les modèles. Les psychothérapeutes appliquent donc, avec souplesse et plus ou moins de fidélité, les modèles connus et maîtrisés et font régulièrement des emprunts à d'autres modèles selon les lectures faites, les séminaires et ateliers de formation auxquels ils participent. Ces emprunts sont parfois faits en toute connaissance de cause et 
parfois à leur insu lorsqu'ils sont en situation difficile. Cependant, on oublie trop souvent que ce n'est pas le modèle qui travaille avec le patient, mais le psychothérapeute. Celui-ci est une personne humaine travaillant au meilleur de sa connaissance, avec ses humeurs, les événements de réalité surgissant dans sa vie personnelle et professionnelle, avec ses sensibilités et ses taches aveugles, ses réactions et ses inhibitions, ses seuils de tolérance à l'angoisse et à la frustration, ses limites personnelles et ses élans d'empathie, etc. C'est pourquoi la supervision ou l'intervision est si essentielle dès que le psychothérapeute travaille auprès de patients sévères, difficiles et régressés.

Bref, n'importe quel modèle psychothérapique doit être appliqué avec intelligence, davantage avec l'intelligence affective que cognitive et avec modération. Il ne faut pas oublier que c'est la qualité de la relation établie entre le psychothérapeute et le patient qui fait l'essentiel du travail. Cependant, pour garantir que cette relation conserve sa qualité psychothérapique il y a des théories qu'il faut bien comprendre, des modèles à bien maîtriser et la supervision professionnelle qui aident à démêler les situations.

\author{
André Renaud \\ 1180, Charles-Albanel \\ Québec (Québec) G1X 4T9 \\ renauda@videotron.ca
}

\title{
Note
}

1. Traduction libre.

\section{Références}

ADLER, G., 1986, Psychotherapy of the narcissistic personality disorder patient : two contrasting approaches, American Journal of Psychiatry, 143, 430-436.

ANZIEU, D., et al., 2003, Les enveloppes psychiques, Dunod, Paris.

BRENNER, C., 1982, The Mind in Conflict, International Universities Press, New York.

Broucek, J. J., 1982, Shame and its relationship to early narcissistic development, International Journal of Psychoanalysis, 63, 369-377.

CLARKIN, J. F., YEOMAN, F. E., KERNBERG, O. F., 1999, Psychotherapy for Borderline Personality, John Wiley and Sons Inc., New York.

CLARKIN, J. F., YEOMAN, F. E., KERNBERG, O. F., 2006, Psychotherapy for Borderline Personality Focusing on Object Relations, American Psychiatric Publishing, Washington, D.C.

FINNEL, J. S., 1985, Narcissistic problems in analysts, International Journal of Psychoanalysis, 66, 433-445.

FREUD, S., 1910, Un souvenir d'enfance de Léonard De Vinci, Euvres complètes, tome X, 19091910, Presses universitaires de France, Paris, 1993, 79-165.

FREUD, S., 1910, Sur la dynamique du transfert, Euvres complètes, Tome XI, 1911-1913, Presses universitaires de France, Paris, 1998, 105-117. 
FREUD, S., 1914, Pour introduire le narcissisme, Euvres complètes, Tome XII, 1913-1914, Presses universitaires de France, Paris, 2005, 213-246.

FREUD, S., 1916-17, La théorie de la libido et le narcissisme, Leçons de psychanalyse, Euvres complètes, Tome XIV, 1915-1917, Presses universitaires de France, Paris, 2000, 427-446.

FREUD, S., 1916, Quelques types de caractères dégagés par le travail psychanalytique, Euvres complètes, Tome XV, 1916-1920, Presses universitaires de France, Paris, 13-41.

GABBARD. G. O., 1994, Psychodynamic Psychiatry in Clinical Practice: the DSM-IV, American Psychiatric Press, Washington, DC.

GABBARD. G. O., 1998, 2000, Transference and countertransference in the treatment of narcissistic patients, in Ronningstam, E. F., 1998, 2000, Disorders of Narcissism; Diagnostic, Clinical, and Empirical Implications, Northvale, N. J., Jason Aronson Inc, 125-146.

HIBBARD, S., 1992, Narcissism, shame, masochism and object relations : an exploratory correlational study, Psychoanalytic Psychology, 9, 489-508.

JACOBSON, E., 1964, Le Soi et le monde objectal, Presses universitaires de France, Coll. Le fil rouge, Paris, 1975.

KERNBERG, O. F., 1970, Factors in the psychoanalytic treatment of narcissistic personalities, Journal of American Psychoanalytic Association, 18, 51-85.

KERNBERG, O. F., 1974, Further contributions to the treatment of narcissistic personalities, International Journal of Psychoanalysis, 55, 215-240.

KERNBERG, O. F., 1975, Borderline Conditions and Pathological Narcissism, Jason Aronson, New York.

KERNBERG, O. F., 1998, 2000, Pathological narcissism and narcissistic personality disorder: Theoretical background and diagnostic classification, in Ronningstam, E. F., ed., Disorders of Narcissism: Diagnostic, Clinical, and Empirical Implications, Jason Aronson Inc, Northvale, N. J., 29-52.

KLEIN, M., 1946, Notes sur quelques mécanismes schizoïdes, in Klein, M., Heimann, P., Issacs, S., Rivière, eds., Développements de la psychanalyse, Presses universitaires de France, Paris, Coll. Bibliothèque de psychanalyse, 274-301, 1966.

KOHUT, H., 1971, Le Soi: La psychanalyse des transferts narcissiques, Presses universitaires de France, Paris, 1974.

KOHUT, H., 1977, The Restoration of the Self, New York, International Universities Press.

KOHUT, H., 1984, How Does Analysis Cure? Goldberg, A., Stepansky, P., eds., University of Chicago Press, Chicago, Illinois.

LEWIN, R. A., SCHULZ, C. G., 1992, Losing and Fusing: Borderline and Transitional Object and Self Relations, J. J. Jason Aronson, Northvale (N.J.).

MANZANO, J., PALACIO ESPASA, F., 2005, La dimension narcissique de la personnalité, Coll. Le Fil Rouge, Presses universitaires de France, Paris.

OGDEN, T. H., 1979, On projective identification, International Journal of Pychoanalysis, 60, 357-373.

OGDEN, T. H., 1982, Projective Identification and Psychotherapeutic Technique, Jason Aronson, New York.

RACKER, H., 1957, The meaning and use of contertransference, Psychoanalytic Quaterly, 26, 303-357.

ROSENFELD, H., 1964, On the psychopathology of narcissism: a clinical approach, International Journal of Psychoanalysis, 45, 332-337.

ROSENFELD, H., 1971, A clinical approach to the psychoanalytic theory of the life and death instincts: an investigation in the aggressive aspects of narcissism, International Journal of Psychoanalysis, 52, 169-178. 
ROSENFELD, H., 1987, Impasse and Interpretation: Therapeutic and Anti-Therapeutic Factors in the Psychoanalytic Treatment of Psychotic, Tavistock, London.

SYMINGTON, N., 1990, The possibility of human freedom and its transmission (with particular reference to the thought of Bion), International Journal of Psychoanalysis, 71, 95-106. WINK, P., 1991, Two faces of narcissism, Journal of Personality and Social Psychology, 61, 590597.

WOLF, E., 1988, Treating the Self: Elements of Clinical Self Psychology, Guilford, New York. 